\title{
Comparison of Low-Cost and Noninvasive Optical Sensors for Cardiovascular Monitoring
}

\author{
Tânia Pereira, Tatiana Oliveira, Manuel Cabeleira, Helena Pereira, Vânia Almeida, \\ João Cardoso, and Carlos Correia
}

\begin{abstract}
New optical probes are developed for carotid distention waveform measurements, in order to assess the risk of cardiovascular diseases. The probes make use of two distinct photodetectors: planar and avalanche photodiodes. Their performance is compared for visible and infrared (IR) light wavelengths. The test setup designed for the evaluation of the probes simulates the fatty deposits commonly seen in the obese people, between skin and the artery. The performed tests show that the attenuation of the signal is lower for the IR light, with higher penetration and better resolution in the captured distension waveform, with higher definition in morphological features on the wave and higher signal-to-noise ratio when compared to the visible source signals. The probes show good overall performance in the test setup with a root mean square error lower than $8 \%$. In vivo, the IR probes allow easier waveform detection, even more relevant with the increasing deposit structures.
\end{abstract}

Index Terms-Biomedical monitoring, optical sensors, photodetectors, silicon radiation detectors.

\section{INTRODUCTION}

$\mathbf{C}$ ARDIOVASCULAR DISEASES (CVD) remain the world's major cause of death [1]. The most important behavioral risk factors of CVDs are unhealthy diet, physical inactivity and tobacco consumption. Often, there are no symptoms of the underlying disease and the first warning usually occurs in the form of a heart attack or a stroke. This emphasizes the need for very early stage diagnostic tools that may be able to detect deviations from normality on parameters that characterize CVDs. The most important cardiovascular risk indicators are: local pulse pressure, the pulse wave velocity and the pulse wave analysis.

Obesity is associated with numerous health issues like obstructive sleep apnea and certain cancers, but the major impact is in CVDs [2] which affect more than 1 billion overweight adults. The effects of obesity on cardiovascular health and disease are many, one of the most profound of

Manuscript received March 16, 2012; revised July 16, 2012; accepted December 18, 2012. Date of publication December 25, 2012; date of current version March 26, 2013. This is an expanded paper from the IEEE SENSORS 2011 Conference. The associate editor coordinating the review of this paper and approving it for publication was Prof. Gerald Gerlach.

The authors are with the Physics Department, Instrumentation and Electronics Group, University of Coimbra, Coimbr 3004 516, Portugal (e-mail: taniapereira@lei.fis.uc.pt; tatianaferreiraoliveira@gmail.com; cabeleira.t.m@ gmail.com; cpereira@lei.fis.uc.pt; vaniagalmeida@gmail.comd; joao.mr. cardoso@gmail.com; correia@lei.fis.uc.pt).

Color versions of one or more of the figures in this paper are available online at http://ieeexplore.iee.org.

Digital Object Identifier 10.1109/JSEN.2012.2236549 which is hypertension. Risk estimates from population studies suggest that more than $75 \%$ of hypertension causes can be directly accounted to obesity [3], [4]. In this context it is necessary that the devices developed for hemodynamic parameters assessment in cardiovascular diseases avoid the difficulties associated with the capturing of the signal in patients with obesity characteristics.

The activity of the cardiac and arterial functions is reflected as changes of diameter (i.e., distension) of the central arteries [5]. During the heart cycle, a pressure wave travels across the arterial tree in a compliant way forcing the blood vessels to distend elastically according to the pressure wave profile. The pulse pressure waveforms contain specific morphological features related to the cardiac and arterial functions. Consequently, the measurement, analysis and monitoring of the distension waveforms allow a better insight in risk assessment of CVDs.

There are already several methods of non-invasive pulse wave measurement based on different principles and sensors. Historically the first method was the sphygmomanometer which measures the blood pressure, as it is attached to a brachial inflatable cuff connected to an aneroid manometer. The brachial cuff is wrapped around the arm and a stethoscope is placed over the artery for the listening of the Korotkoff sounds, which appear and disappear while the cuff deflates, although this technique only provides information on the maximum and minimum of the pressure [5]. Another technique is the applanation tonometry in which the artery is flattened by applying pressure non-invasively to squeeze the artery against the bone. The applied pressures required to maintain the flattened shape of the probe have to be present during all the recording. This is accomplished by using an array of sensors, each of which measures pressure. This is hard to achieve, since the sensor has to be correctly placed to obtain zero stress of the artery and no deformational stress, which turns applanation tonometry an operator mastery dependent technique [6]. In the last years, piezoelectric based probes have been widely used in arterial pressure waveform assessments, which generate a measurable voltage at the output contacts if they are mechanically deformed [7], [8], and [9]. The photoplethysmography is another available solution, based on the transmission of the infrared light produced by a light emitting diode (LED) through a finger or earlobe, which is detected by a photodetector, producing an attenuation profile volume waveform from which changes in volume can be measured. The gold standard for the assessment of the carotid 

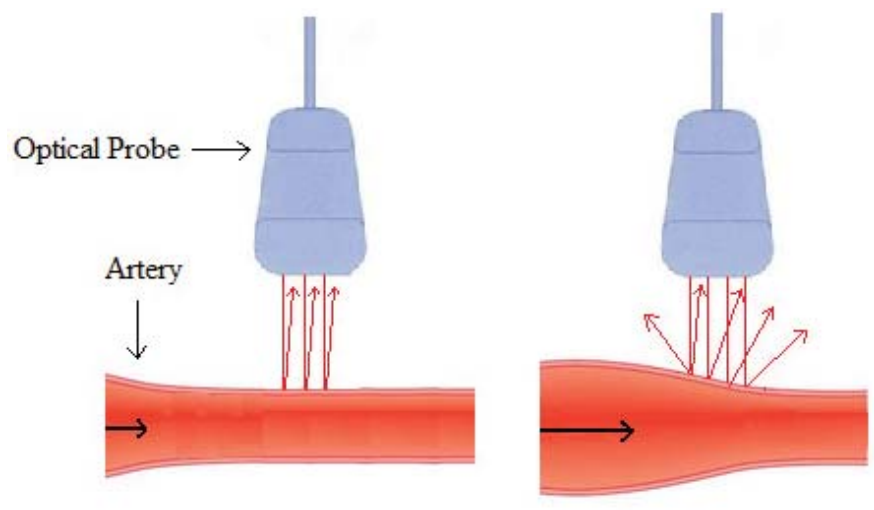

Fig. 1. Effect on the reflectivity with a passing pressure pulse.

distension waveform is the ultrasonography, which is an established source of clinical and experimental information [10]. The ultrasound system measures the intensity of the echo providing information about the type of structures in the scanning region. The technology is especially accurate for delineating the interface between solid and fluid filled spaces, which is a key requirement for vascular analysis [11], [12].

The different methods allow measurements of different parameters on the pulse pressure however, none of them are free of interference on the signals detected by requiring mechanical contact, and the commercial systems have limitations on the number of parameters determined.

The distensions in vessel walls, generated by a pressure wave, travel across the arterial tree and can be optically measured in the peripheral arteries like the carotid, as they run very close to the surface and impart a measurable distension. This distension, as it modulates the reflection characteristics of the skin or the artery itself, can be used to generate an optical signal correlated with the passing pressure wave (Fig. 1). Optical sensors are an attractive instrumental solution in this kind of time assessment applications due to their truly non-contact operation capability, which ensures a contactless measurement [11].

The performance of the optical probes was evaluated in comparison with a cardiovascular ultrasound system, the gold standard for assessment of the carotid distension waveform. The optical sensors allow the reproduction of the arterial waveform with a higher resolution, adequate to feed feature extraction algorithms [11].

In the case of obese people, the detection of the pressure wave with the existing methods is rather difficult or impossible. The applanation tonometry in obese subjects is very difficult, as this technique needs a rigid structure to sustain the artery and therefore, fatty structures between the artery and the bone should be avoided [6].

Situations where the carotid artery is not near the skin surface require wavelengths able to optically penetrate the tissues in order to allow the determination of the distension waveform. To fulfill those needs, probes fitted with infrared (IR) illumination were developed, in addition to the ones using visible light.

The penetration depth of light into a biological tissue is an important parameter for the correct determination of the signal detected by the photodetectors. The near-infrared region of the electromagnetic spectrum provides a window of opportunity with greater tissue penetration. In the $700-1000 \mathrm{~nm}$ wavelengths range the tissue penetration is on the order of 10-15 mm [13]. The normal carotid arteries lie $10 \mathrm{~mm}$ beneath the skin [14].

As in the plethysmography principle, in this optical system the pulsatile changes in the light transmission through the living tissues occur due to the changes in the arterial blood volume on that tissue. The measurement of the pulsatile component would eliminate the variations of the light absorption by the tissues, skin, pigment, etc. Thus, only the pulsatile absorbance between the light source and the photodetector corresponds to the pulsatility of the arteries in the resultant signal [15].

The main absorber in skin tissue is the melanin pigment, a highly effective absorber of light, particularly in the UV and visible wavelength ranges. Most of the scattering in blood is also due to the red blood cells, although there are also small contributions from the leucocytes, the platelets and from the large albumin molecules. The carotid tissue is mainly composed of elastin and collagen fibers and, as expected, it is very thick and consequently responsible for the large scattering and reflection of the light [16].

The current study describes the development and optimization process of four (4) optical probes, used for the measurement of the distension profile, over time, at the carotid artery site. All the probes have a common design structure which includes the LED illumination in the vicinity of the photodiode sensor. Both elements (light source and sensor) are integrated in the probe's printed circuit board (PCB) which also includes basic electronics conditioning [17], [18], and [19].

\section{INSTRUMENTATION}

Throughout the development process, the probes were designed to more effectively detect the signal (providing the sensor in the center position and the LED evenly), as show in Prototype Probe Head in Fig. 2. The PCB is cased in a plastic box and the elements form an ergonomic configuration to ensure a safe and user-friendly probe for both operator and patient.

Silicon photodiodes are semiconductor devices able to response to high energy particles and photons. Photodiodes operate by absorption of photons or charged particles and generate a current flow in an external circuit, proportional to the incident light intensity.

Two distinct types of silicon optical sensors are used in this work: planar (PPD) and avalanche photodiode (APD), each one requiring a particular electronic circuitry. By choosing the correct semiconductor material and the correct casing it is possible to make a sensor capable of generating a photocurrent related only to the spectra of interest.

The photodetectors used in this work are not sensitive to the angle of incidence of the light, integrating the number of photons per unit area and converting them into electric current. The illumination is provided by two LEDs for each photodetector disposed in an axial symmetry. The LEDs approximately 


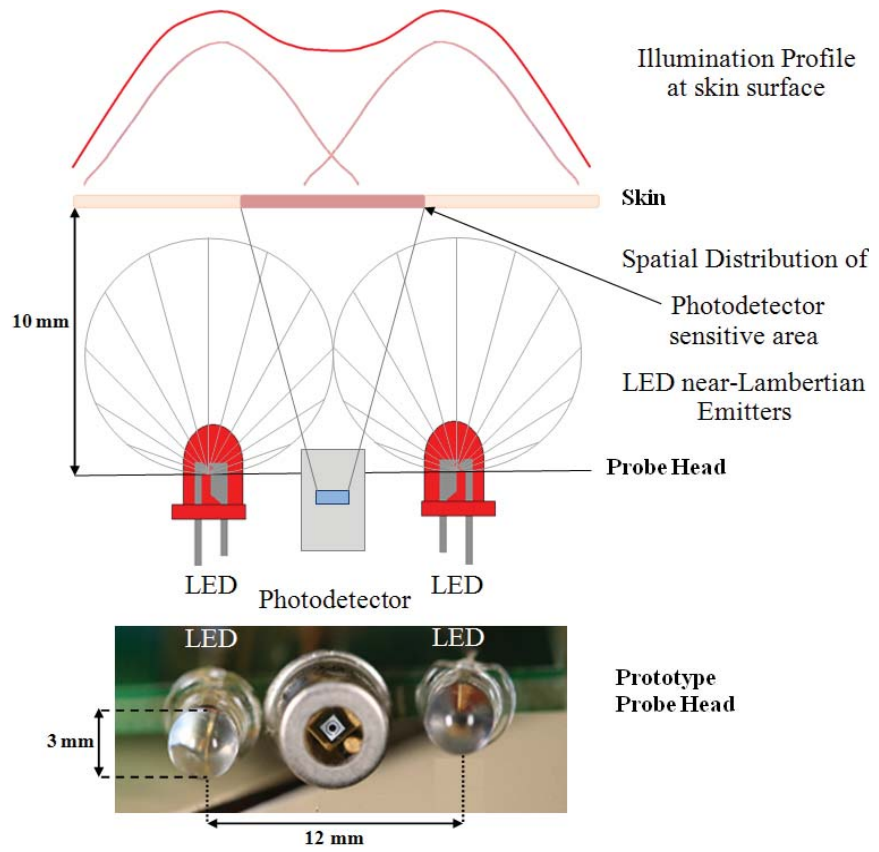

Fig. 2. Structure of the optical probe with APD photodiode sensor and visible light (red) sources.

act as a point source of light, but have an angular light distribution, as represented in the Illumination Profile at skin surface of Fig. 2, and, because the skin is a diffuse surface a homogeneous distribution of the reflected light is ensured. Thus, a uniform illumination scheme is provided in the area (about $140 \mathrm{~mm}^{2}$ ) where the reflection occurs, neglecting the influence of the light incidence angle.

A silicon photodiode can be operated in either the photovoltaic or photoconductive mode. Mode selection depends upon the speed requirements of the application, and the amount of dark current that is tolerable. In this case the photodiodes were operated in the photoconductive mode in order to achieve their fastest switching speeds. The electronic circuit is composed by op-amps for a current to voltage conversion (trans-impedance amplifier).

The spectral response curve represents the absolute responsivity as a function of the wavelength. This curve is commonly shown as a relative response curve in relation to its peak (highest responsivity). The response of the silicon photodiode to the wavelength of the incident light is between $400 \mathrm{~nm}$ to $1100 \mathrm{~nm}$, for both the PPD and APD. The responsivity is low at the short wavelengths, increasing until the maximum sensitivity wavelength is reached $(940 \mathrm{~nm}$ for the PPD and $830 \mathrm{~nm}$ for APD) and decreases thereafter.

\section{A. Planar Photodiode}

The photodiode is a device in which the electric field of the depletion layer of a $\mathrm{P}-\mathrm{N}$ junction is used to collect current carriers generated by photonic absorption. The diffused area defines the photodiode active area. The electron-hole pairs generated by light incidence are swept away by drifting in the depletion region and are collected by diffusion from the undepleted region. The generated current is proportional to the incident light or radiation power.
The responsivity of a silicon photodiode is a measure of the sensitivity to light. It is a measure of the effectiveness of the conversion of the light intensity into electrical current, which depends on the wavelength of the incident light. In this work a planar type from Silonex (SLCD-61N3), rectangularshaped photodiode, $10.2 \mathrm{~mm} \times 5.1 \mathrm{~mm}$, biased at $15 \mathrm{~V}$ on the photoconductive mode, with a maximum sensitivity wavelength for $940 \mathrm{~nm}$, was used and, consequently, the LEDs selected for this probe have also this wavelength.

\section{B. Avalanche Photodiode}

An avalanche photodiode is a photodiode that internally amplifies the photocurrent by an avalanche process. In APDs, a large reverse-bias voltage, typically over 150 Volts, is applied across the active region. The high voltage causes the photoelectrons, initially generated by the incident radiation to accelerate as they move through the APD active region. As these electrons collide with other electrons in the semiconductor material, they cause a fraction of them to become part of the photocurrent. This process is known as avalanche multiplication. Avalanche multiplication continues to occur until the electrons move out of the active area of the APD. Under these conditions, gains of around 50 will result from the avalanche effect, providing a larger signal from small variations of light reflected from the skin and will, at least theoretically, improve the signal-to-noise ratio (SNR).

APDs used in the probes (Advanced Photonics (SD 012-70-62-541)) have a sensitive maximum to light in the infrared wavelength region, specifically at $830 \mathrm{~nm}$. $T S$ Series (Matsusada) is used for a high voltage DC-to-DC converter suited for biasing APDs.

\section{Skin Emulation Model}

The different tissues that compose the skin (essentially fatty deposits and muscle) have particular optical properties. The indices of light absorption and reflection for each structure allow the understanding of the penetration depth of each wavelength, from the skin to the carotid artery.

There is an optical window in the skin on the region of 600-1300 nm. Wavelengths below $600 \mathrm{~nm}$ are strongly absorbed by proteins, melanin, acids and DNA while infrared wavelengths can easily cross the skin and fat barrier before reaching the carotid artery [20].

In this work, LED light wavelengths capable of crossing the epidermis (melanin layer) and fat structures that could eventually exist, up to its reflection only at the carotid artery were selected. The ideal optic window is in the IR wavelength.

For obese subjects, independently of the cardiovascular condition, acquiring signals at the carotid site using optical sensors can be a hard task to accomplish. In this sense, the use of IR light allows tissue penetration and captures the distension waveform deeper than in the case of visible light that is mostly reflected at the skin surface level (Fig. 3).

Silicone rubber has optical and mechanical properties similar to those of the skin and is often used to build phantoms in human tissue optical studies [21]. For this reason, silicone layers were used in the developed test bench in order to 


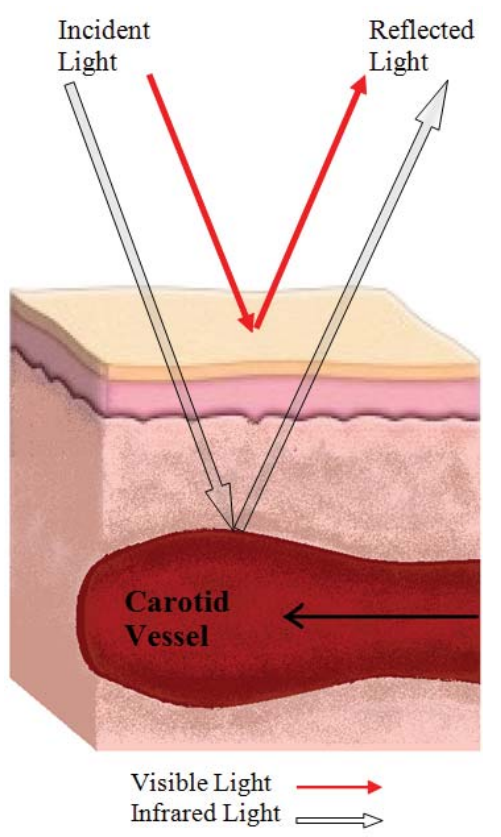

Fig. 3. Structure at the carotid site and its interaction with radiation.

simulate the thickness of the structures that make up the skin and fatty accumulations.

The optical absorption measured across the wavelength range of 300 to $1700 \mathrm{~nm}$, shows that the silicone has strong absorption bands in the mid-infrared spectrum range, with a maximum at $1260 \mathrm{~nm}$, and has a high refractive index and a lower absorption $(<1 \%)$ in the visible spectrum [22], [23].

This study compares three different wavelengths, 635, 830 and $940 \mathrm{~nm}$ and two photodetectors (APD and PPD) for the assessment of the distension waveform in the carotid.

\section{TEST SETUP}

The test setup was designed to evaluate the optical ability of the developed probes in measuring distensions with the same magnitude of the ones observed at the carotid site. Different number of silicone layers was placed in front of the probes, to simulate the fat structures that can be accumulated in the neck, between the skin and the artery.

The arbitrary waveform generator (AWG), Agilent 33220A, in Fig. 4, was previously loaded with typical cardiac waveforms, as described in the literature [24], which feeds an actuator, (ACT), $700 \mu \mathrm{m}$ Physik Instrumente GmbH, P-287, driven by a high voltage linear amplifier (HV Amp), Physik Instrumente GmbH, E-508.

The cardiac waveforms generated in the test setup were of two types, one corresponding to a healthy individual, type C and one with pathological features, type A [24]. The actuator describes this waveforms and moves a mechanical structure (MS) separated from the probe by a variable number of static layers of silicone rubber.

In the probe, light is produced by two LEDs (L1 and L2). The lighting system uses a current regulator based on transistors to ensure uniform target illumination. Variations of the reflected light are detected in the photodiode

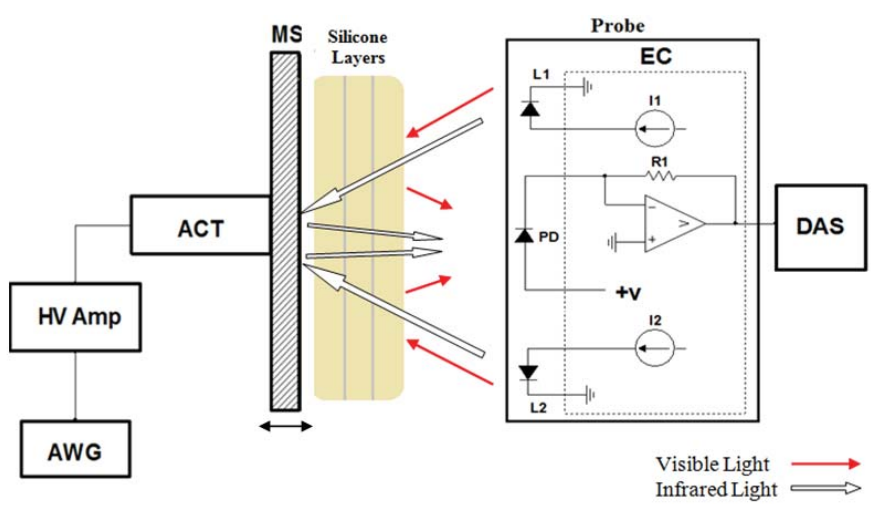

Fig. 4. Schematic of test setup. Light interaction with silicone layers and photo detection circuit.

and driven to dedicated electronic circuitry (EC), which complete the optical probe as a whole.

The signals were acquired using a 16-bit resolution data acquisition system (National Instruments, USB6210) with a sampling rate of $20 \mathrm{kHz}$ and stored for offline analysis using Matlab.

\section{REsults}

On the test setup the signals from the two types of probes (PPD and APD) were acquired with several silicone layers to simulate different levels of fat accumulation. Each silicone layer has a $1 \mathrm{~mm}$ thickness and the tests were conducted using 2 to 10 layers $(10 \mathrm{~mm})$. The signals were acquired for cardiac shaped pulses of frequencies $0.5,1$ and $1.5 \mathrm{~Hz}$, to ensure that healthy and pathological cardiac frequencies were covered. This range was chosen to enclose possible heart rate at rest, normal conditions in the acquisition of signals.

Signal acquisition was made in human carotid arteries in order to compare the results obtained with the analysis in the test setup.

\section{A. Signals in the Test Setup}

The signals were obtained for both red and infrared lights, transmitted through two, four, six, eight and ten silicone layers. Fig. 5 shows the waveform, type $\mathrm{C}$ with $1 \mathrm{~Hz}$, detected by the PPD with different number of silicon layers.

The reflected radiation that reaches the photodetector is composed by the light reflected on the surface of the silicone and by the component that is reflected in the deeper layers. The reflection from the top surface corresponds to the DC component of the signal detected and the AC component captures the reflected light at the pulsating mechanical structure in movement. The DC component was removed and the AC component was normalized. The main objective of these probes is the measurement of the pressure waveform, and the layers have a low pass filter effect that do not affected the waveform profile but decreases the signal amplitude and the SNR level.

The intensity of the transmitted light decreases exponentially with the increasing number of layers, i.e., the thickness of the homogeneous body. Lambert's law (1) describes the 


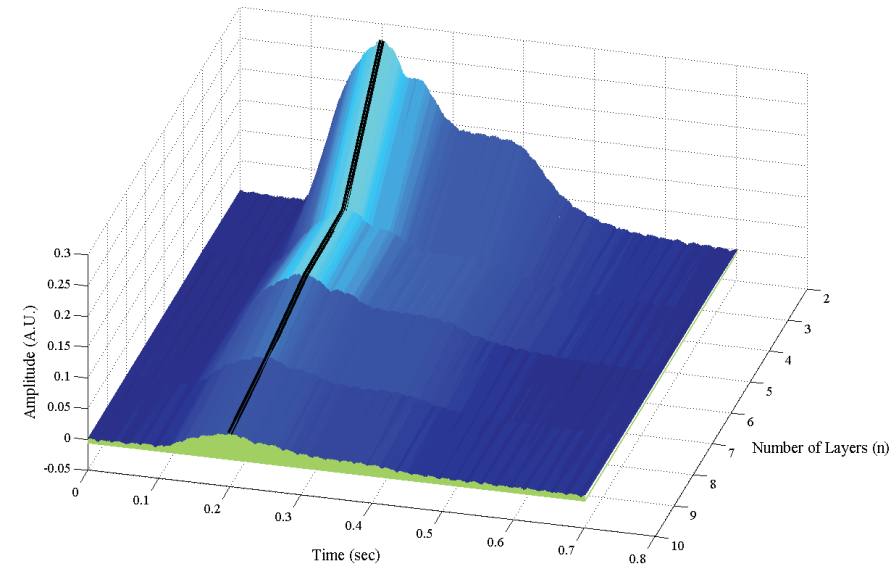

Fig. 5. PPD signals with visible light for different number of silicone layers and exponentially attenuation (black line).

amount of attenuation (absorption) that the radiation undergoes passing through a thickness of material, where $\mu$ is the linear absorption (or attenuation) coefficient

$$
I=I_{0} e^{-\mu n} .
$$

In (1), the measured intensity $I$ transmitted through a silicone layer with thickness $n$ is related to the incident intensity $I_{0}$ according to the inverse exponential power law.

The attenuation coefficient is a quantity that characterizes how easily a material can be penetrated by the light. A large attenuation coefficient means that the beam is quickly "attenuated" as it passes through the material, and a small attenuation coefficient means that the material is relatively transparent to the beam. The attenuation coefficient was determined for the two probes with visible and infrared light.

The black line, in Fig. 5, is the attenuation suffered by the detected radiation with the increasing number of silicone layers. Using an exponential fit to this curve, it is possible to determine the attenuation coefficient for each wavelength, visible and infrared light. For visible light this attenuation coefficient is $\mu_{\text {visible }}=0.5930 \mathrm{~mm}^{-1}$ and for infrared light is $\mu_{\mathrm{IR}}=0.3971 \mathrm{~mm}^{-1}$. The augmentation of the radiation absorbed by the silicone layers and the decreasing of the transmitted light that reaches the photodetector are evident in the increasing of the computed attenuation coefficient. For the APD probe with visible or infrared light the behavior is similar. For the signals obtained in the test bench, a spectral analysis was performed in order to determinate the attenuation provided by the silicone layers. Fig. 6 shows the spectrum obtained for signals acquired with the avalanche photodetector with visible light, without silicone (grey line) and with 10 layers (black line).

In the frequency window of interest for the analyzed signal, 40 to $100 \mathrm{~Hz}$, there is a nearly constant attenuation at about $20 \mathrm{~dB}$ for signals acquired with ten silicone layers. It is possible to identify in the spectrum the frequencies which correspond to the electronic interference detected by the optical sensors, such as the frequencies of the light bulbs $(50 \mathrm{~Hz})$ or monitors used during the acquisition $(87 \mathrm{~Hz})$. The silicone

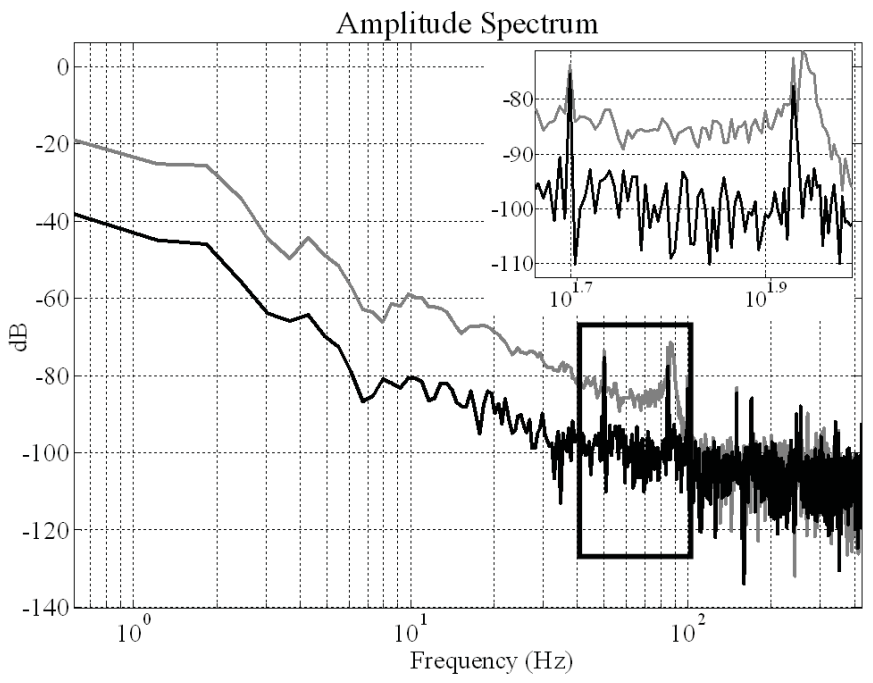

Fig. 6. Amplitude spectrum for APD signals with visible light without silicone layers (grey) and with 10 silicone layers (black).

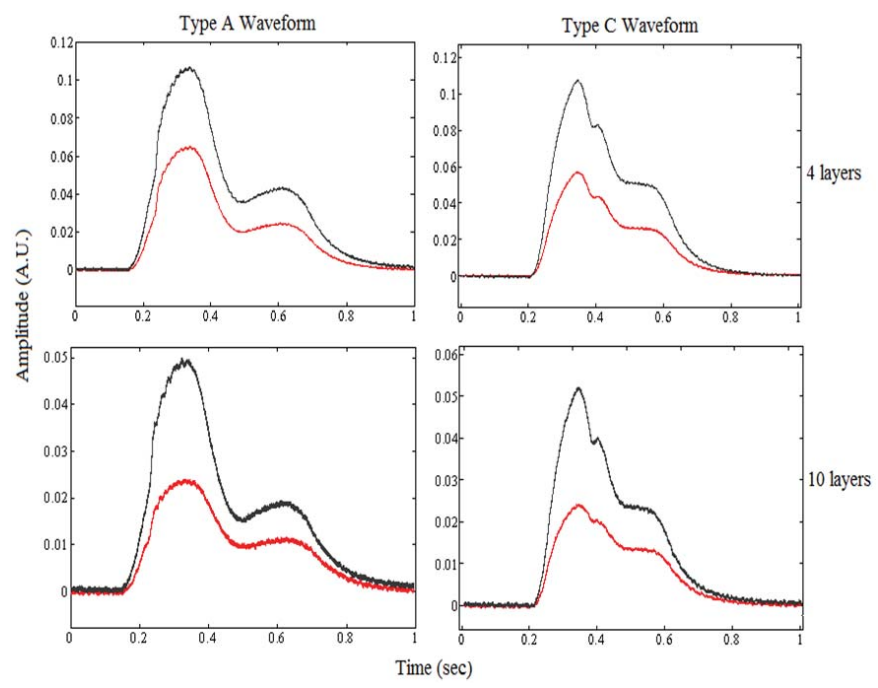

Fig. 7. APD signals for four and ten layers, with visible (red line) and IR light (grey line).

attenuating those two frequencies in the transmitted optical signal just the same, but the electrical pickup from electronics devices is overwhelming the optical signal. For the IR light, the attenuation also occurs at low frequencies with smaller impact, at an almost constant attenuation level of less than $10 \mathrm{~dB}$.

In the case of the planar probe, the spectral analysis of the acquired signals showed the same attenuation at low frequencies ( $20 \mathrm{~dB}$ for of visible light and $10 \mathrm{~dB}$ for IR light).

The signals from four and ten silicone layers for waveform types $\mathrm{A}$ and $\mathrm{C}$ acquired at $1 \mathrm{~Hz}$ are shown in Fig. 7. The signals acquired by the APD probe with infrared light, exhibit higher amplitude as well as a better waveform resolution. The signals acquired with visible light, marked in red, show smaller signal amplitude and a higher noise interference imparting lower resolution to the waveform, mainly in the morphological features that enclose a great clinical importance. 


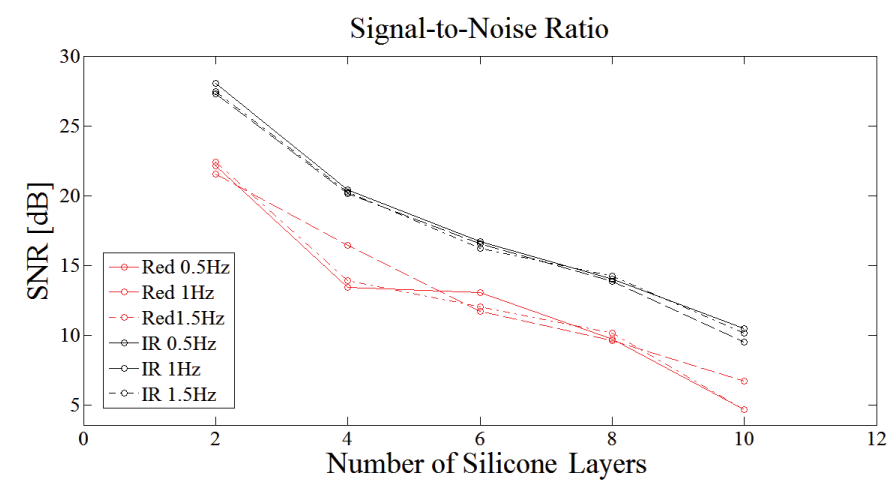

Fig. 8. SNR for PPD probes with different numbers of silicone layers.

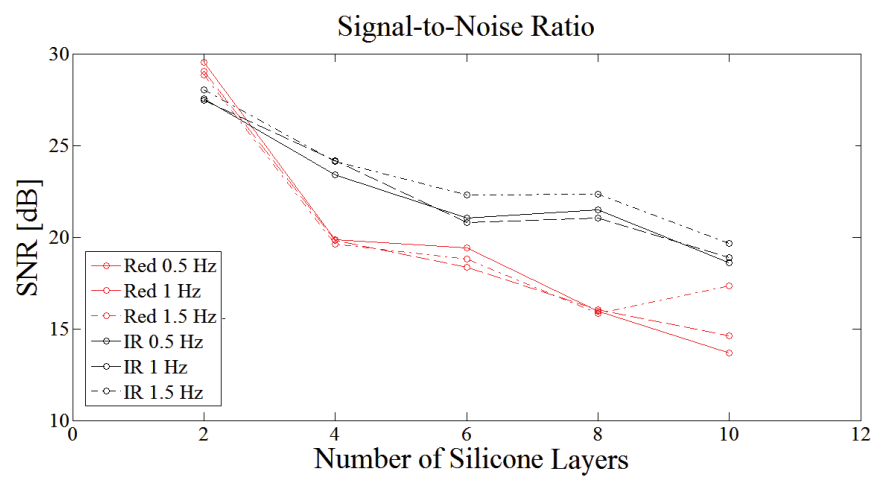

Fig. 9. SNR for APD probes with different number of silicone layers.

Once again, IR light exhibits a better performance, with the lower RMSE values, although there is not a clear distinction between the photodetectors used.

Another parameter that is used to quantify how much a signal has been corrupted by noise is the SNR. Its value in $\mathrm{dB}$ is computed by the relation between signal amplitude and noise amplitude. The experimental determination of this parameter was accomplished by acquiring signals from the photodetector with the movement of the actuator and in static conditions. The last one yields just the noise since there is no signal while the first is the signal plus noise component. Results from the two probes for $\mathrm{A}$ and $\mathrm{C}$ waveform types for different frequencies are shown in Fig. 8.

The SNR from the PPD probes for the red and infrared wavelengths decrease with the increment of the number of silicone layers. It is also noticeable that the SNR obtained with infrared light is always greater than its counterpart obtained with red light (Table I). SNR values for APD probes are shown in Fig. 9. For two silicone layers, (low fat accumulation), visible and IR light perform similarly. However, as the number of silicone layers increases, IR light probe show higher SNR values.

\section{B. In-Vivo Carotid Measurements}

Following the preliminary tests with both systems, a set of in-vivo carotid measurements were carried out to validate the use of the optical probes for in vivo conditions.

The radiation spectrum of a person is the same as the one of a blackbody. The Wien's Law allows the determination
TABLE I

MAXimum RMS ERror BetweEn the Signals From the WAVEForm Generator and the Signals Detected From Each Probe

\begin{tabular}{|c|c|c|}
\hline & Visible & IR \\
\hline PPD & $7.19 \%$ & $6.13 \%$ \\
\hline APD & $7.95 \%$ & $5.66 \%$ \\
\hline
\end{tabular}

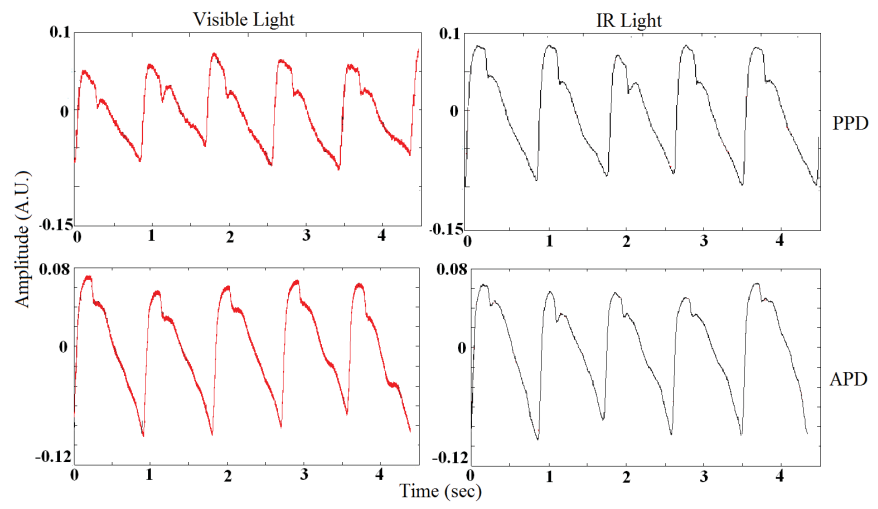

Fig. 10. PPD and APD signals obtained at the human carotid using visible (red line) and infrared light (grey line).

of the maximum wavelength of radiation for a human (body temperature of $37^{\circ} \mathrm{C}$ ), that is $9700 \mathrm{~nm}$. This wavelength corresponds to the infrared radiation, also known as body heat. This wavelength is well above the electromagnetic spectrum range used by the optical probes, as the spectral range of sensitivity of both, the planar and avalanche photodiodes, is between 400 and $1100 \mathrm{~nm}$. The influence of the infrared radiation emitted as animal heat, in this specific case the human body, is negligible [25].

The study protocol was approved by the ethical committee of the Coimbra Central Hospital (CHC), Portugal. All subjects were volunteers and gave a written informed consent. The waveform was measured in the carotid artery by a near contact placement. Measurements were performed while subjects were in a quiet environment after at least 10 minutes of supine rest and the signals were stored for offline analysis.

In the acquired signal, the DC component represents the light absorption of the tissue, venous blood, and non-pulsatile arterial blood, and the signal has a baseline drift caused by the patient's natural movements (such as breathing). The AC component of the signal is related to the pulsatile arterial blood.

The resulting waveforms were obtained along several cardiac cycles. The signals obtained in the human carotids show that the infrared probes allow the acquisition of cardiac waveforms with lower noise and, consequently, better definition at the morphological features (Fig. 10). Another, not so obvious, advantage of the IR lit probes is that it eases the operator's task since the detection of the optimal positioning of the probe becomes faster. This effect assumes great importance in practice since it can determine the overall time spend in acquiring data from a patient.

\section{CONCLUSION}

The developed optical probes demonstrated a good overall performance on the test setup system. The probes with infrared 
light show better results with the progressive increase in the number of silicone layers. They also provide better resolution in the waveform features and higher SNR and lower RMSE when compared to the signals acquired with visible light.

The APD has an inner gain of 50 due to its intrinsic avalanche effect and is considered almost point-like $\left(1 \mathrm{~mm}^{2}\right)$. On the other hand, the PPD has a detection area of $52 \mathrm{~mm}^{2}$, which is 52 times larger than the APD detection area. However, as the PPD does not have any inherent gain, we can consider that the gain of the APD compensates the detection area of the PPD and thus, the signals can be compared. APD probes exhibit a better performance for both visible and IR lighting, with better SNR when compared to the signals from the PPD probes. On the other hand, the APD has a stronger electric field which allows the decreasing of the drift velocity, a fast response time and an increased bandwidth response.

The study of the attenuation coefficient showed that there is a greater absorption of visible light, when compared to the infrared light, in the silicon layers for both the APD and PPD probes: $\mu_{\text {Visible }}>\mu_{\mathrm{IR}}$. For the probes with visible illumination, a lower amount of light is transmitted than with infrared illumination, and the signal detected is poorer, demonstrating the preference for the ability of the infrared light in tissue penetration, in order to detect the signal in cases of fat accumulation.

Spectral analysis of the signals obtained in the test setup showed that the silicone layers attenuated about $20 \mathrm{~dB}$ for the signals acquired by the optical probes in the case of visible light, but less than $10 \mathrm{~dB}$ in the case of infrared light.

In human carotids, the infrared probes allow an easier waveform detection of the signal in the photodiode. This effect becomes even more relevant with the increasing of the fatty structures thickness, which is accumulated in the obese neck.

The good results obtained with the PPD combining with the much lower cost of the PPD detector than the APD and the easy bias voltage of $15 \mathrm{~V}$, does the solution LED/PPD combination the best option.

In the future, the primary optimization is the development of a simpler method for acquisition and processing of the signals, as it needs to be performed by an expert operator. The simplification of the process will allow an easier use of the probes in the clinical environment.

\section{ACKNOWLEDGMENT}

The authors acknowledge the support from Fundação para a Ciência e Tecnologia (FCT) for funding (PTDC/SAUBEB/100650/2008). Project developed under the initiative of QREN, funding by UE/FEDER, through COMPETE Programa Operacional Factores de Competitividade.

\section{REFERENCES}

[1] The Top Ten Causes of Death, World Health Organization, Geneva, Switzerland, Feb. 2007.

[2] C. J. Lavie, R. V. Milani, and H. O. Ventura, "Obesity and cardiovascular disease: Risk factor, paradox, and impact of weight loss," J. Amer. Colloq. Cardiol., vol. 53, no. 21, pp. 1925-1932, 2009.

[3] R. M. Krauss, M. Winston, B. J. Fletcher, and S. M. Grundy, "Obesity: Impact on cardiovascular disease," Circulation, vol. 98, pp. 1472-1476, May 1998.
[4] B. Mathew, L. Francis, A. Kayalar, and J. Cone, "Obesity: Effects on cardiovascular disease and its diagnosis," J. Amer. Board Fam. Med., vol. 21, no. 6, pp. 562-568, Nov.-Dec. 2008.

[5] A. P. Avolio, M. Butlin, and A. Walsh, "Arterial blood pressure measurement and pulse wave analysis-their role in enhancing cardiovascular assessment," Physiol. Meas., vol. 31, no. 1, pp. 1-47, 2009.

[6] I. S. Mackenzie, I. B. Wilkinson, and J. R. Cockcroft, "Assessment of arterial stifness in clinical practice," QJM, Int. J. Med., vol. 95, no. 2 , pp. 67-74, 2002.

[7] H. C. Pereira, T. Pereira, V. Almeida, E. Borges, E. Figueiras, J. B. Simões, J. L. Malaquias, J. M. Cardoso, and C. M. Correia, "Characterization of a double probe for local pulse wave velocity assessment," Physiol. Meas., vol. 31, no. 11, pp. 1449-1465, 2010.

[8] J. McLaughlin, M. McNeill, B. Braun, and P. D. McCormack, "Piezoelectric sensor determination of arterial pulse wave velocity," Physiol. Meas., vol. 24, no. 3, pp. 693-702, 2003

[9] V. Almeida, H. C. Pereiraa, T. Pereiraa, E. Figueirasa, E. Borgesa, J. M. R. Cardosoa, and C. Correiaa, "Piezoelectric probe for pressure waveform estimation in flexible tubes and its application to the cardiovascular system," Sens. Actuators A, Phys., vol. 169, no. 1, pp. 217-226, Sep. 2011.

[10] A. Reisner, P. A. Shaltis, D. McCombie, and H. H. Asada, "Utility of the photoplethysmogram in circulatory monitoring," Anesthesiology, vol. 108, no. 5, pp. 950-958, 2008.

[11] T. Pereira, T. Oliveira, M. Cabeleira, P. Matos, H. C. Pereira, V. Almeida, E. Borges, H. Santos, T. Pereira, J. Cardoso, and C. Correia, "Signal analysis in a new optical pulse waveform profiler for cardiovascular applications," in Proc. Signal Image Process. Appl., vol. 716. Sipa, Greece, Jun. 2011, pp. 19-25.

[12] J. G. Webster, Encyclopedia of Medical Devices and Instrumentation, New York: Wiley, 1988.

[13] U. Mahmood and R. Weissleder, "Near-Infrared optical imaging of proteases in cancer," Molecular Cancer Therapeutics, vol. 2, pp. 489-496, May 2003.

[14] A. Nicolaides, K. W. Beach, E. Kyriakou, and C. S. Pattichis, Ultrasound and Carotid Bifurcation Atherosclerosis. New York: Springer-Verlag, 2012.

[15] V. Kamat, "Pulse oximetry," Indian J. Anaesth, vol. 46, no. 2, pp. 261-268, 2002.

[16] P. van der Zee, "Measurement and modelling of the optical properties of human tissue in the near infrared," Ph.D. dissertation, Dept. Med. Phys. Bioeng., Univ. College London, London, U.K., Dec. 1992.

[17] T. Pereira, M. Cabeleira, P. Matos, E. Borges, H. C. Pereira, V. Almeida, J. Cardoso, and C. Correia, "Optical methods for local pulse wave velocity assessment," in Proc. 4th Int. Joint Conf. Biomed. Eng. Syst. Technol., Rome, Italy, 2011, pp. 74-81.

[18] T. Pereira, M. Cabeleira, P. Matos, E. Borges, H. C. Pereira, V Almeida, J. Cardoso, and C. Correia, "Non-contact pulse wave velocity assessment using optical methods," in Biomedical Engineering Systems and Technologies (Communications in Computer and Information Science), vol. 273. Berlin, Germany: Springer-Verlag, 2013, pp. 246-257.

[19] T. Pereira, T. Oliveira, M. Cabeleira, V. Almeida, E. Borges, J. Cardoso, C. Correia, and H. C. Pereira, "Visible and infrared optical probes for hemodynamic parameters assessment," in Proc. IEEE Sensors Conf., Oct. 2011, pp. 28-31.

[20] A. Bashkatov, E. Genina, V. Kochubey, and V. Tuchin, "Optical properties of human skin, subcutaneous and mucous tissues in the wavelength range from 400 to $2000 \mathrm{~nm}$," J. Phys. D, Appl. Phys., vol. 38, no. 15, pp. $2543-2555,2005$

[21] A. Gherardi, "A skin surface characterization system based on capacitive image analysis," Ph.D. dissertation, ARCES, Università di Bologna, Ciudad De Buenos Aires, Argentina, Dec. 2007.

[22] J. DeGroot, A. Norris, S. Glover, and T. Clapp, "Highly transparent silicone materials," Proc. SPIE, Linear Nonlinear Opt. Organic Mater. $I V$, vol. 5517, pp. 116-123, Oct. 2004

[23] B. Riegler and R. Thomaier, Optical Silicones for Use in Harsh Operating Environments. Philadelphia, PA: AMS, Oct. 2004.

[24] M. D. Melis, U. Morbiducci, E. R. Rietzschel, M. D. Buyzere, A. Qasem, L. V. Bortel, T. Claessens, F. M. Montevecchi, A. Avolio, and P. Segers "Blood pressure waveform analysis by means of wavelet transform," Med. Biol. Eng. Comput., vol. 47, no. 2, pp. 165-173, 2009.

[25] I. P. Herman, Physics of the Human Body, New York: Springer-Verlag, 2007. 
Tânia Pereira was born in 1986. She received the M.S. degree in biomedical engineering from the University of Coimbra, Coimbra, Portugal, in 2009. She is currently pursuing the Ph.D. degree in biomedical engineering with the University of Coimbra.

Her current research interests include development of noninvasive instrumentation based in different optical sensors for the assessment of cardiovascular parameters for diagnosis and monitoring of diseases.

Tatiana Oliveira was born in 1988. She received the Master degree in biomedical engineering from the University of Coimbra, Coimbra, Portugal, in 2011.

Her current research interests include the development of noninvasive instrumentation for hemodynamic parameters assessment for risk evaluation and diagnosis and on the development of biomedical devices for ambient assisted living monitoring.

Manuel Cabeleira was born in 1986. He received the M.S. degree in biomedical engineering from the University of Coimbra, Coimbra, Portugal, in 2010 .

He is a Researcher with the Physics Department, University of Coimbra. His current research interests include the development of new methods of calibrating Cochlear implants based in eye-tracking techniques.

Helena Pereira received the Academic degree in biomedical engineering from the University of Coimbra, Coimbra, Portugal, in 2007. She is currently pursuing the Ph.D. degree with the same university, for the research and development of novel instrumentation for hemodynamic parameters assessment.

She has been a Staff Member of ISA, since 2007, where she has responsibility over several projects related to remote vital signs and ambient assisted living. Her current research interests include PET and later in the development of sensors and other biomedical devices.
Vânia Almeida was born in 1986. She received the MS degree in Biomedical Engineering from Coimbra University, Portugal in 2009. She is currently pursuing the $\mathrm{PhD}$ degree in Department of Physics at University of Coimbra.

Her current research involves development of non-invasive sensors and instrumentation to evaluate cardiovascular diseases. Her research interest includes biomedical signal processing, embedded software development on real time, and biomedical circuit design.

João Cardoso received the Ph.D. degree on the research area of digital pulse processing techniques for nuclear spectrometry in 2006.

$\mathrm{He}$ is a Research Scientist with the Instrumentation Center, University of Coimbra, Coimbra, Portugal. His current research interests include optimization of instrumentation for physics experiments in the fields of astrophysics and medium energy particle physics as well as the development of innovative instruments and sensors for biomedical engineering.

Carlos Correia received the Ph.D. degree in physics and his studies to signal processing, electronics and biomedical instrumentation in 1985.

He has been a Full Professor with the Physics Department, University of Coimbra, Coimbra, Portugal, since 2000. He has over 100 published works in the literature. 